\title{
A comparative study on design optimization of polygonal and Bézier curve-shaped thin noise barriers using dual BEM formulation
}

\author{
Rayco Toledo, Juan J. Aznárez, Orlando Maeso and David Greiner
}

\begin{abstract}
The inclusion of sound barriers for abating road traffic noise is a broadly used strategy that is often constrained by the requirements associated with its effective height. Due to this fact, the searching process has to deal with compromise solutions between the effective height and the acoustic efficiency of the barrier, assessed by the insertion loss (IL) in this paper. Two different barrier designs are studied herein for two different receivers configurations and for three clearly distinguishable regions in terms of closeness to the barrier. These models are based on the optimization of the IL of thin-cross section profiles proposed by an Evolutionary Algorithm. The special nature of these sort of barriers makes necessary the implementation of a dual BEM formulation in the optimization process. Results obtained show the usefulness of representing complex thin-cross section barrier configurations as null boundary thickness-like models.
\end{abstract}

\section{Introduction}

The inclusion of sound barriers for abating the negative effects of road traffic noise near residential areas is a broadly used strategy. Considerable research work and studies focused on sound diffraction around barriers have been carried out in the past two decades, specifically in the prediction of the performance and the development of more efficient designs. Amongst all of the different theoretical methods proposed concerning the issue, the Boundary Element Method (BEM hereinafter) has been previously used by the authors of this work [6] in the analysis of complex barrier configurations.

Rayco Toledo, Juan J. Aznárez, Orlando Maeso and David Greiner

Instituto de Sistemas Inteligentes y Aplicaciones Numéricas en Ingeniería (SIANI), Edificio Central del Parque Científico y Tecnológico del Campus Universitario de Tafira, 35017 Las Palmas de G.C., Spain, e-mail: rtoledo, jaznarez, omaeso, dgreiner@iusiani.ulpgc.es 
Evolutionary Algorithms (EA) have been widely used for Shape Design Optimization problems in numerous Engineering fields. The combined use of optimization problems using EA with a BEM code has been implemented in sound barriers design problems within the institute where this work is developed (see [2], [4] and [3]).

The Insertion Loss coefficient (IL henceforward) is a valuable estimator to assess the acoustic efficiency of the barrier. This parameter represents the sound pressure level difference in the situation with and without the presence of the barrier at a particular point (receiver). Whilst the parameters involving the efficiency are numerous, the effective height of the barrier $\left(h_{\text {eff }}\right)$ is the factor with greatest influence. Constraints related to this factor force the searching process to find compromise solutions between the effective height and the acoustic efficiency of the barrier. These profiles generally feature complex configurations and its implementation in optimization processes is often found to be difficult in terms of validating its topological feasibility. Two different barrier designs are studied in this work. These models are based on the optimization of the acoustic efficiency of thin-cross section profiles, idealized as null boundary thickness, proposed by an EA. The special nature of these sort of barriers makes necessary the implementation of a dual BEM formulation in the optimization process.

\section{Modelling and discretization by implementing a dual BEM formulation}

The next lines are devoted to the implementation of a dual BEM formulation in thin noise barriers idealized as null boundary thickness profile (see Fig. 1). The special nature of these sort of barriers makes necessary the addition of a complementary formulation (hyper-singular) that coupled with the conventional BEM formulation yields a compatible system of equations.

\subsection{Singular BEM formulation}

The integral equation for the $i$ boundary point to be solved by the singular BEM formulation can be expressed as follows:

$$
c_{i} p_{i}+f_{\Gamma_{b}} p \frac{\partial p^{*}}{\partial \mathbf{n}_{j}} d \Gamma=p_{0}^{*}+\int_{\Gamma_{b}} \frac{\partial p}{\partial \mathbf{n}_{j}} p^{*} d \Gamma
$$

This integral equality just involves the boundary of the barrier under investigation. The $f$ symbol represents the integral along the boundary to be understood in the Cauchy principal value sense, once the singularity around the collocation point $i$ has been extracted $\left(c_{i}\right)$. In Ec. (1), $p$ is the acoustic pressure field over the barrier surface and $p^{*}$ is the half-space fundamental solution (the acoustic pressure field 
when the source is placed at the collocation point $i$ over a plane with admittance $\beta_{g}$ (ground admittance)) and $c_{i}$ is the free term. On the whole: $c_{i}=\theta / 2 \pi$, where $\theta$ represents the inner angle to the boundary measured in radians. It is easily shown that $c_{i}=0.5$ for smooth boundaries.

The expressions of the fundamental solution and its derivative for a perfectly reflective ground for bi-dimensional, harmonious problems are:

$$
\begin{aligned}
p^{*}(k, r) & =\frac{1}{2 \pi}\left[K_{0}(i k r)+K_{0}(i k \bar{r})\right] \\
\frac{\partial p^{*}}{\partial n} & =-\frac{i k}{2 \pi}\left[K_{1}(i k r) \frac{\partial r}{\partial \mathbf{n}_{j}}+K_{1}(i k \bar{r}) \frac{\partial \bar{r}}{\partial \mathbf{n}_{j}}\right]
\end{aligned}
$$

being $i$ the imaginary unit, $k$ the wave number, $r$ and $\bar{r}$ the distances from the source and the image point to the observation point respectively, and $K_{0}$ and $K_{1}$ the Bessel modified functions of order 0 and 1 respectively.

By discretizing the boundary, the integral kernels of the fundamental solution of the singular BEM formulation are yielded:

$$
h_{k}^{i j}=\int_{\Gamma_{j}} \frac{\partial p^{*}}{\partial \mathbf{n}_{j}} \phi_{k} d \Gamma_{j} \quad ; \quad g_{k}^{i j}=\int_{\Gamma_{j}} p^{*} \phi_{k} d \Gamma_{j}
$$

A system of equations is obtained from this process and leads to values of acoustic pressure on the barrier boundary.

\subsection{Hyper-singular BEM formulation}

The integral equation for the $i$ boundary point to be solved by the hyper-singular BEM formulation can be written as follows:

$$
c_{i}\left(\frac{\partial p_{i}}{\partial \mathbf{n}_{i}}\right)+f_{\Gamma} p \frac{\partial^{2} p^{*}}{\partial \mathbf{n}_{i} \partial \mathbf{n}_{j}} d \Gamma=f_{\Gamma} \frac{\partial p^{*}}{\partial \mathbf{n}_{i}} \frac{\partial p}{\partial \mathbf{n}_{j}} d \Gamma+\frac{\partial p_{0}^{*}}{\partial \mathbf{n}_{i}}
$$

where the $f$ and $f$ symbols represent the integral along the boundary to be understood in the Hadamard finite part integral and in the Cauchy principal value sense, respectively. The hyper-singular formulation of the method demands that the source placement (collocation point $i$ ) be inside the element (non-nodal collocation point) (see [8]). Thus, in (4) it is satisfied that $c_{i}=0.5$.

Expression (5) shows the values of the fundamental solution and its derivative for the hyper-singular formulation: 


$$
\begin{aligned}
\frac{\partial p^{*}}{\partial \mathbf{n}_{i}} & =-\frac{i k}{2 \pi}\left[K_{1}(i k r) \frac{\partial r}{\partial \mathbf{n}_{i}}+K_{1}(i k \bar{r}) \frac{\partial \bar{r}}{\partial \mathbf{n}_{I}}\right] \\
\frac{\partial^{2} p^{*}}{\partial \mathbf{n}_{i} \partial \mathbf{n}_{j}} & =\frac{(i k)^{2}}{2 \pi}\left[\left(K_{2}(i k r) \frac{\partial r}{\partial \mathbf{n}_{i}} \frac{\partial r}{\partial \mathbf{n}_{j}}+\frac{K_{1}(i k r)}{r} \mathbf{n}_{i} \cdot \mathbf{n}_{j}\right)+\left(K_{2}(i k \bar{r}) \frac{\partial \bar{r}}{\partial \mathbf{n}_{I}} \frac{\partial \bar{r}}{\partial \mathbf{n}_{j}}+\frac{K_{1}(i k \bar{r})}{\bar{r}} \mathbf{n}_{I} \cdot \mathbf{n}_{j}\right)\right]
\end{aligned}
$$

Similarly to Ec. (2), $i$ is the imaginary unit, $k$ the wave number and $r, \bar{r}$ the distances to the observation point from the collocation point and its symmetric point with respect to the ground plane, respectively. It is worth making a distinction here regarding the normal vectors involved in the expressions above. $\mathbf{n}_{j}$ is the normal to the boundary at the integration point and $\mathbf{n}_{i}\left(n_{x}^{i}, n_{y}^{i}\right), \mathbf{n}_{I}\left(n_{x}^{i},-n_{y}^{i}\right)$ represent the normal vectors to the real boundary at the collocation point $(i)$ and at its symmetric point $(I)$ placed on a fictitious, symmetric boundary with respect to the ground plane, respectively. $K_{1}$ and $K_{2}$ represent the Bessel modified functions of order 1 and 2, respectively.

After a discretization process along the boundary, expression (4) yields a numerical solution from which the integral kernels of the hyper-singular BEM formulation are obtained, for $i$ collocation point when integrated over $j$ element:

$$
m_{k}^{i j}=\int_{\Gamma_{j}} \frac{\partial^{2} p^{*}}{\partial \mathbf{n}_{i} \partial \mathbf{n}_{j}} \phi_{k} d \Gamma_{j} \quad ; \quad l_{k}^{i j}=\int_{\Gamma_{j}} \frac{\partial p^{*}}{\partial \mathbf{n}_{i}} \phi_{k} d \Gamma_{j}
$$

The numerical resolution of these integrals deserve a thorough treatment and can be consulted in [8] and [7].

\subsection{Dual BEM formulation}

Fig. 1 represents a generic thin-cross section noise barrier to be solved by dual BEM formulation. After a discretization process, each node holds the values of pressure and flux with respect to the boundary normal, i.e., both at the left and at the right according to the direction of travel on the boundary $\left(p^{+}, q^{+}, p^{-}, q^{-}\right.$hereinafter).

Fig. 1 (a) Idealization of a generic thin-cross section noise barrier profile as null thickness boundaries. (b) Strategy used to avoid the singularity around the collocation point in BEM formulation.

(a)

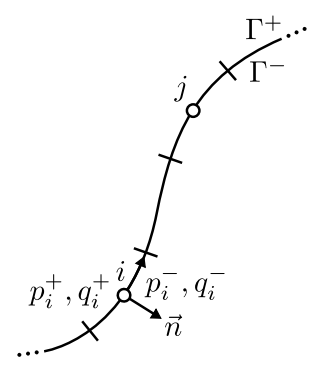

(b)

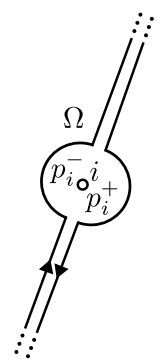


Fig. 1(b) represents the strategy used to isolate the singularity of the method in this sort of domains. Thus, the matrix equality of the singular BEM formulation for thin-cross section noise barriers can be expressed as follows:

$$
c_{i}\left(p_{i}^{+}+p_{i}^{-}\right)+\sum_{j=1}^{N}\left(\mathbf{H}_{j}^{+} p_{j}^{+}+\mathbf{H}_{j}^{-} p_{j}^{-}\right)=\sum_{j=1}^{N}\left(\mathbf{G}_{j}^{+} q_{j}^{+}+\mathbf{G}_{j}^{-} q_{j}^{-}\right)
$$

being $N$ the overall nodes number of the discretization over the boundary. Considering that $\mathbf{n}^{+}=\mathbf{n}^{-}$at the collocation point $j$, it is easily shown that:

$$
\mathbf{H}_{j}^{+}=-\mathbf{H}_{j}^{-} \quad ; \quad \mathbf{G}_{j}^{+}=\mathbf{G}_{j}^{-}
$$

For internal noise sources and smooth boundaries, the final expression can be written as follows (see [5]):

$$
\left(\frac{1}{2}\right) \Sigma p_{i}+\sum_{j=1}^{N} \mathbf{H}_{j}^{+} \Delta p_{j}=\sum_{j=1}^{N} \mathbf{G}_{j}^{+} \Sigma q_{j}+p_{0}^{*}
$$

where:

$$
\Sigma p_{i}=p_{i}^{+}+p_{i}^{-} ; \Delta p_{j}=p_{j}^{+}-p_{j}^{-} \quad ; \Sigma q_{j}=q_{j}^{+}+q_{j}^{-}
$$

Deriving (7) with respect to $\mathbf{n}_{i}^{+}$an integral equality of the hyper-singular BEM formulation is obtained:

$$
c_{i}\left(\frac{\partial p_{i}^{+}}{\partial \mathbf{n}_{i}^{+}}+\frac{\partial p_{i}^{-}}{\partial \mathbf{n}_{i}^{+}}\right)+\sum_{j=1}^{N}\left(\mathbf{M}_{j}^{+} p_{j}^{+}+\mathbf{M}_{j}^{-} p_{j}^{-}\right)=\sum_{j=1}^{N}\left(\mathbf{L}_{j}^{+} q_{j}^{+}+\mathbf{L}_{j}^{-} q_{j}^{-}\right)
$$

where:

$$
\frac{\partial p_{i}^{-}}{\partial \mathbf{n}_{i}^{+}}=-q_{i} \quad ; \quad \mathbf{M}_{j}^{+}=-\mathbf{M}_{j}^{-} \quad ; \quad \mathbf{L}_{j}^{+}=\mathbf{L}_{j}^{-}
$$

The hyper-singular formulation of the method requires that the collocation point $i$ be inside the element (see [8]) what assurances that the inner angle to the boundary at that point is always $\theta=\pi$. In this way, the final dual BEM expressions for internal noise sources for both the singular and hyper-singular formulation are:

$$
\begin{aligned}
\left(\frac{1}{2}\right) \Sigma p_{i}+\sum_{j=1}^{N} \mathbf{H}_{j}^{+} \Delta p_{j} & =\sum_{j=1}^{N} \mathbf{G}_{j}^{+}\left(A^{+} \Sigma p_{j}+A^{-} \Delta p_{j}\right)+p_{0}^{*} \\
\left(\frac{1}{2}\right)\left(A^{-} \Sigma p_{i}+A^{+} \Delta p_{i}\right)+\sum_{j=1}^{N} \mathbf{M}_{j}^{+} \Delta p_{j} & =\sum_{j=1}^{N} \mathbf{L}_{j}^{+}\left(A^{+} \Sigma p_{j}+A^{-} \Delta p_{j}\right)+\frac{\partial p_{0}^{*}}{\partial \mathbf{n}_{i}}
\end{aligned}
$$


being:

$$
\begin{aligned}
& \Delta q_{j}=A^{-} \Sigma p_{j}+A^{+} \Delta p_{j} ; \Sigma q_{j}=A^{+} \Sigma p_{j}+A^{-} \Delta p_{j} \\
& A^{+}=-(1 / 2) i k\left(\beta^{+}+\beta^{-}\right) ; A^{-}=-(1 / 2) i k\left(\beta^{+}-\beta^{-}\right)
\end{aligned}
$$

Finally, expression (13) can be expressed matricially as:

$$
\left[\begin{array}{cc}
\frac{\mathbf{I}}{2}-\mathbf{G}^{+} A^{+} & \mathbf{H}^{+}-\mathbf{G}^{+} A^{-} \\
\frac{A^{-}}{2} \mathbf{I}-\mathbf{L}^{+} A^{+} & \frac{A^{+}}{2}
\end{array}\right]\left[\begin{array}{c}
\Sigma p \\
\mathbf{I}+\mathbf{M}^{+}-\mathbf{L}^{+}-A^{-}
\end{array}\right]=\left[\begin{array}{c}
p_{o}^{*} \\
\frac{\partial p_{o}^{*}}{\partial \mathbf{n}_{i}^{+}}
\end{array}\right]
$$

\section{Problem definition}

Fig. 2 represents the general configuration of the model under study. It deals with a two-dimensional model concerning an infinite, coherent mono-frequency source of sound, placed parallel to an infinite noise barrier of thin cross-section that stands on a flat plane (ground) of uniform admittance at $d_{s}=10 \mathrm{~m}$. Both the ground and the barrier feature a perfectly reflective surface in this article $\left(\beta_{g}=\beta_{b}=0\right)$. A trapezoidal section holds the area for feasible profiles, defined by the barrier projection to the ground, that is constant and $\mathrm{d}_{\mathrm{p}}=1 \mathrm{~m}$, and the maximum effective height to be achieved, that is $h_{\text {eff }}=3 \mathrm{~m}$ at the median of the rectangle trapezium.

Table 1 Data concerning regions under study.

\begin{tabular}{ccccccc}
\hline Region & $\mathrm{d}_{\mathrm{s}}(\mathrm{m})$ & $\mathrm{d}_{\mathrm{p}}(\mathrm{m})$ & $\mathrm{d}_{\mathrm{r}_{1}}(\mathrm{~m})$ & $\mathrm{d}_{\mathrm{r}_{2}}(\mathrm{~m})$ & $\Delta \mathrm{x}(\mathrm{m})$ & $\Delta \mathrm{y}(\mathrm{m})$ \\
\hline 1 & & & 0.5 & 10.0 & 2.0 & 1.0 \\
2 & \multirow{2}{*}{10.0} & \multirow{2}{*}{1.0} & 10.5 & 40.0 & 8.0 & 2.0 \\
3 & & & 50.5 & 50.0 & 10.0 & 5.0 \\
\hline
\end{tabular}

Two different receiver configurations are studied. In one configuration (Ca) a group of four receivers placed on the ground and separated $\Delta \mathrm{x}$ from one another is considered. In the other configuration $(\mathrm{Cb})$, four groups of four receivers are studied. The first group is laid on the ground and the remaining ones are placed at different heights, separated among them by a distance of $\Delta y$. In accordance with the former configuration, the horizontal distance among the receivers of a group is $\Delta \mathrm{x}$.

In addition to this, three clearly distinguishable regions in terms of closeness to the median of the feasible region $\left(\mathrm{d}_{\mathrm{r}_{1}}\right)$ are proposed for both receiver configurations. Table 1 holds the data concerning these regions.

The results achieved are given in terms of insertion loss (IL), defined as follows: 


$$
\mathrm{IL}=-20 \log _{10}\left(\frac{\mathrm{P}_{\mathrm{B}}}{\mathrm{P}_{\mathrm{HS}}}\right)(\mathrm{dB})
$$

on every frequency of the band spectra, and represents the sound pressure level at the receiver points as a difference between the situation with $\left(\mathrm{P}_{\mathrm{B}}\right)$ and without $\left(\mathrm{P}_{\mathrm{HS}}\right)$ considering the barrier. This parameter is a widely used estimator to assess the acoustic efficiency of sound barriers.

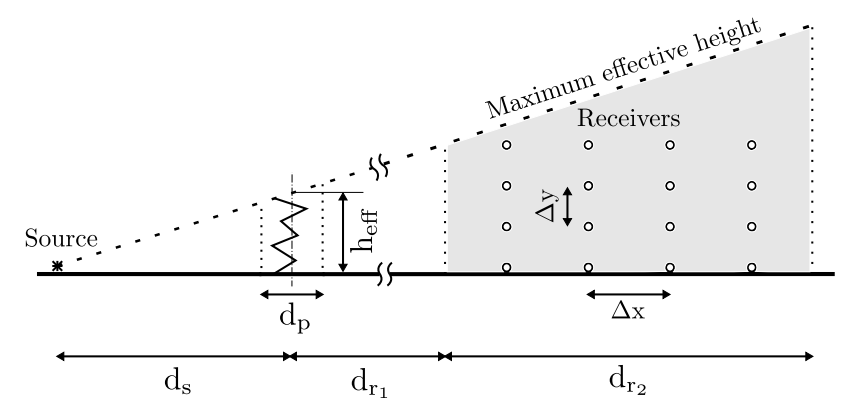

Fig. 2 Two-dimensional configuration for thin cross-section acoustic barriers.

\section{Methodology}

This section provides an overview of the proposed methodology for the optimization of thin-cross section noise barriers idealized as null boundary thickness-like models.

\subsection{Shape optimization}

Shape design optimization is carried out by the combined use of an EA and a code that implements a dual BEM formulation. The EA software used in this work applies the GAlib package [9]. This library is a collection of $\mathrm{C}++$ genetic algorithm (GA) components from which it is possible to quickly construct GA's to attack a wide variety of problems.

In this paper, a steady-state genetic algorithm is used replacing the two worst individuals (in terms of their fitness function) at every generation, with a population size of 100 individuals. A single-point crossover operator is used in this study, with a crossover rate of 0.9 . The considered mutation rate is $1 / n_{c h}$, where $n_{c h}$ is the chromosome length $\left(\mathrm{n}_{\mathrm{ch}}=8 \mathrm{xn}\right.$, being $\mathrm{n}$ the overall number of the design variables -of 8 bits precision each-). Five independent executions of the optimization process are considered for each model and configuration. The stop-criterion condition is met for 20,000 evaluations of the fitness function (FF). 
A transformed domain is considered (see [3]). This domain holds the set of design variables of the model under study, denoted by $\left(\xi_{i}, \eta_{i}\right)$, and represents the rectangular search space for the GA (see left part of Fig. 3). Every $\left(\xi_{i}, \eta_{i}\right)$ point in the transformed domain has its image $\left(x_{i}, y_{i}\right)$ in the Cartesian space, that is the real domain where the barrier operates. In this paper $h_{\text {eff }}=3 \mathrm{~m}$ is proposed. This generates a trapezoidal search space in the Cartesian barrier domain (see right part of Fig. 3).

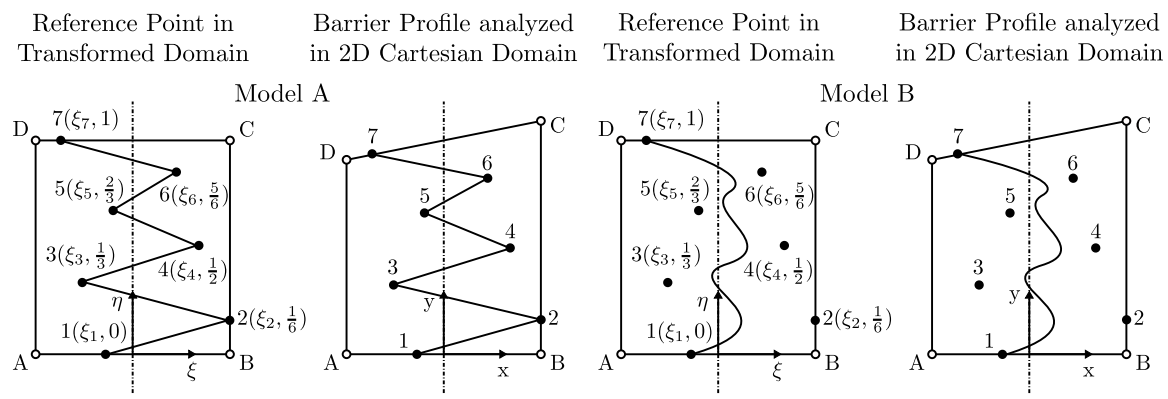

Fig. 3 Design variables and models under study.

Two acoustic barrier designs are studied along this paper (Fig. 3). The horizontal projection $\left(\mathrm{d}_{\mathrm{p}}\right)$ and the effective height $\left(\mathrm{h}_{\mathrm{eff}}\right)$ are identical for each design. Both models are built from seven points, being the first and the last one on the ground and on the effective height line respectively. The vertical distance among the points is $d_{i}=1 / 6$ in the search space (transformed domain) and they are just allowed to feature horizontal movements. Model A is a polygonal curve-shaped barrier built from points through which straight slopes pass. Model B is a $6^{\text {th }}$ degree Bézier curve-shaped barrier built from seven control points of which only the first (0) and the last (7) belong to it.

\subsection{Assessment of the insertion loss}

Taking into account the overall value of the IL of the frequency band spectrum analyzed for each receiver seems to be a more realistic estimator to evaluate the efficiency of a sound barrier. Consequently, (17) represents the average IL value for each frequency and receiver when using the ISO 717.2 normalized traffic noise spectra for third-octave band center frequencies [1], ranging from 100 to 2,000 Hz.

$$
\overline{\mathrm{IL}}=-10 \cdot \log _{10}\left(\frac{\sum_{\mathrm{i}=1}^{\mathrm{NF}} 10^{\left(\mathrm{A}_{\mathrm{i}}-\mathrm{IL}_{\mathrm{i}}\right) / 10}}{\sum_{\mathrm{i}=1}^{\mathrm{NF}} 10^{\mathrm{A}_{\mathrm{i}} / 10}}\right)(\mathrm{dBA})
$$


being NF the studied spectra number of frequencies, i.e. $N F=14, A_{i}$ the spectra A-weighted noise level and $\mathrm{IL}_{\mathrm{i}}$ the insertion loss value for sources pulsing at every frequency of the spectra.

\subsection{Definition of the fitness function}

Shape optimization is carried out entirely based on the overall IL mean value of all receiver points.

$$
\mathrm{FF}=\sum_{\mathrm{j}=1}^{\mathrm{NR}} \overline{\mathrm{IL}}_{\mathrm{j}} / \mathrm{NR}
$$

being $\overline{\mathrm{IL}}_{\mathrm{j}}$ the IL mean value for each receiver (see (18)) and NR the total number of receivers. This value corresponds to the fitness function $(\mathrm{FF})$ to be maximized, so the higher its value the higher the acoustic efficiency of the sound barrier.

\section{Results and Discussion}

Tables 2 and 3 collect the acoustic efficiency and the coordinates of the design variables (see Fig. 3) of the best individuals respectively, for each receiver configuration, region and model.

Fig. 4 and 5 show the barrier profile of the best individuals of the models under study, in terms of its acoustic efficiency as well as the average frequential IL evolution of the receivers for each region and model.

Fig. 6 and 7 show the evolution of the average of the fitness function $\left(\mathrm{FF}_{\text {Average }}\right)$, the best individual $\left(\mathrm{FF}_{\mathrm{Best}}\right)$ and the average of the standard deviation for both models under study and every receiver configuration.

In the light of the results the following analysis is carried out:

- The polygonal-shaped barrier outperforms the acoustic efficiency of the $6^{\text {th }}$ degree Bézier curve-shaped model for the near region when the receivers are placed on the ground (Ca configuration). However, the latter model performs a better acoustic behaviour for non-near regions (over $1 \mathrm{dBA}$ ).

- Both models under study display similar acoustic performances when a grid of receivers is considered in the shadow region of the barrier, with the exception of the intermediate region in which the Bézier model outperforms the polygonal design in half a decibel.

- According to the comparative analysis between the optimized models and the straight barrier the need to study designs alternative to the latter is suggested, even for far regions.

- Model B displays a wider variety among best individuals of the population than in the case for Model A according to the evolution of the highest standard deviation 
(Fig. 6 and 7), meaning that the convergence of the optimization process turns out to be more cumbersome in Model B (optimization process is easier in Model A).

Table 2 Acoustic efficiency of the models under study.

\begin{tabular}{|c|c|c|c|c|c|c|}
\hline $\mathrm{RC}^{*}$ & Region & Model & $\mathrm{L}_{\mathrm{c}}(\mathrm{m})$ & $\Delta \mathrm{L}_{\mathrm{c}}(\mathrm{m})$ & $\mathrm{FF}_{\text {best }}(\mathrm{dBA})$ & $\Delta \mathrm{FF}_{\text {best }}(\mathrm{dBA})$ \\
\hline \multirow{6}{*}{$\mathrm{Ca}$} & \multirow{2}{*}{1} & A & 4.08177 & +1.08177 & 17.92628 & +4.32966 \\
\hline & & B & 3.60547 & +1.60547 & 16.77495 & +3.17833 \\
\hline & \multirow{2}{*}{2} & A & 3.97839 & +0.97839 & 12.93384 & +1.02711 \\
\hline & & B & 3.51863 & +0.51863 & 14.08611 & +2.17938 \\
\hline & \multirow{2}{*}{3} & A & 3.52785 & +0.52785 & 12.46634 & +1.04716 \\
\hline & & B & 3.84417 & +0.84417 & 13.57048 & +2.15130 \\
\hline \multirow{6}{*}{$\mathrm{Cb}$} & \multirow{2}{*}{1} & A & 4.10065 & +1.10065 & 16.95941 & +2.41822 \\
\hline & & B & 3.41333 & +0.41333 & 16.83553 & +2.29434 \\
\hline & \multirow{2}{*}{2} & A & 3.63842 & +0.63842 & 14.36767 & +0.92615 \\
\hline & & B & 3.71865 & +0.71865 & 14.87088 & +1.42936 \\
\hline & \multirow{2}{*}{3} & A & 3.60034 & +0.60034 & 13.64344 & +0.88584 \\
\hline & & B & 3.68994 & +0.68994 & 13.75215 & +0.99450 \\
\hline
\end{tabular}

${ }^{*}$ Receiver configuration.

Table 3 Design variables of the best individuals.

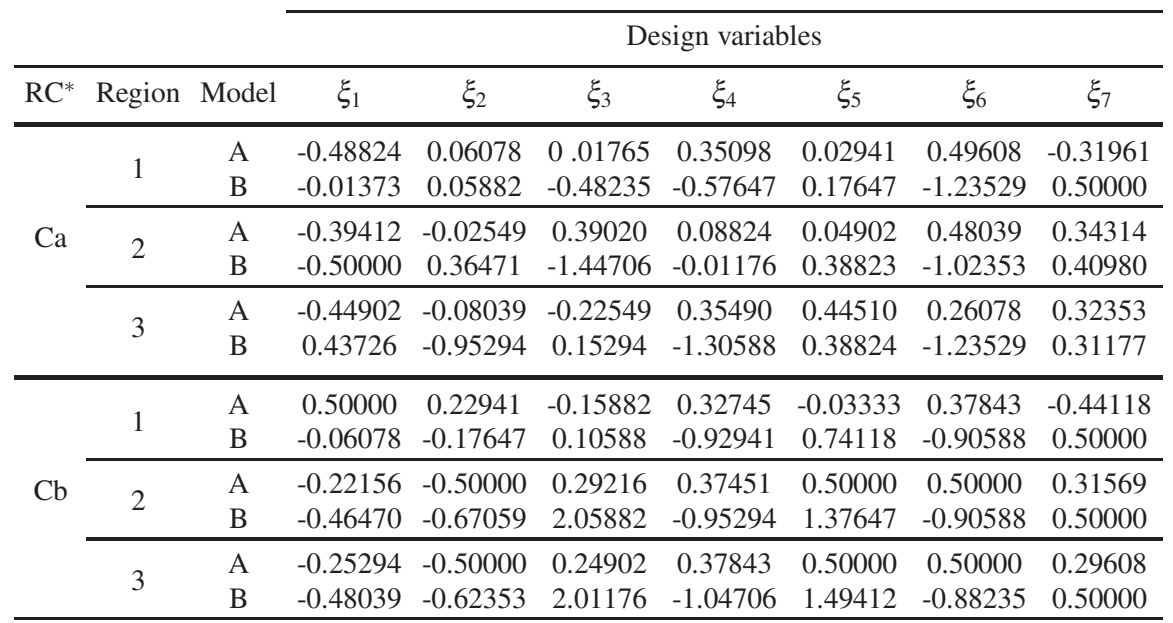

*Receiver configuration. 

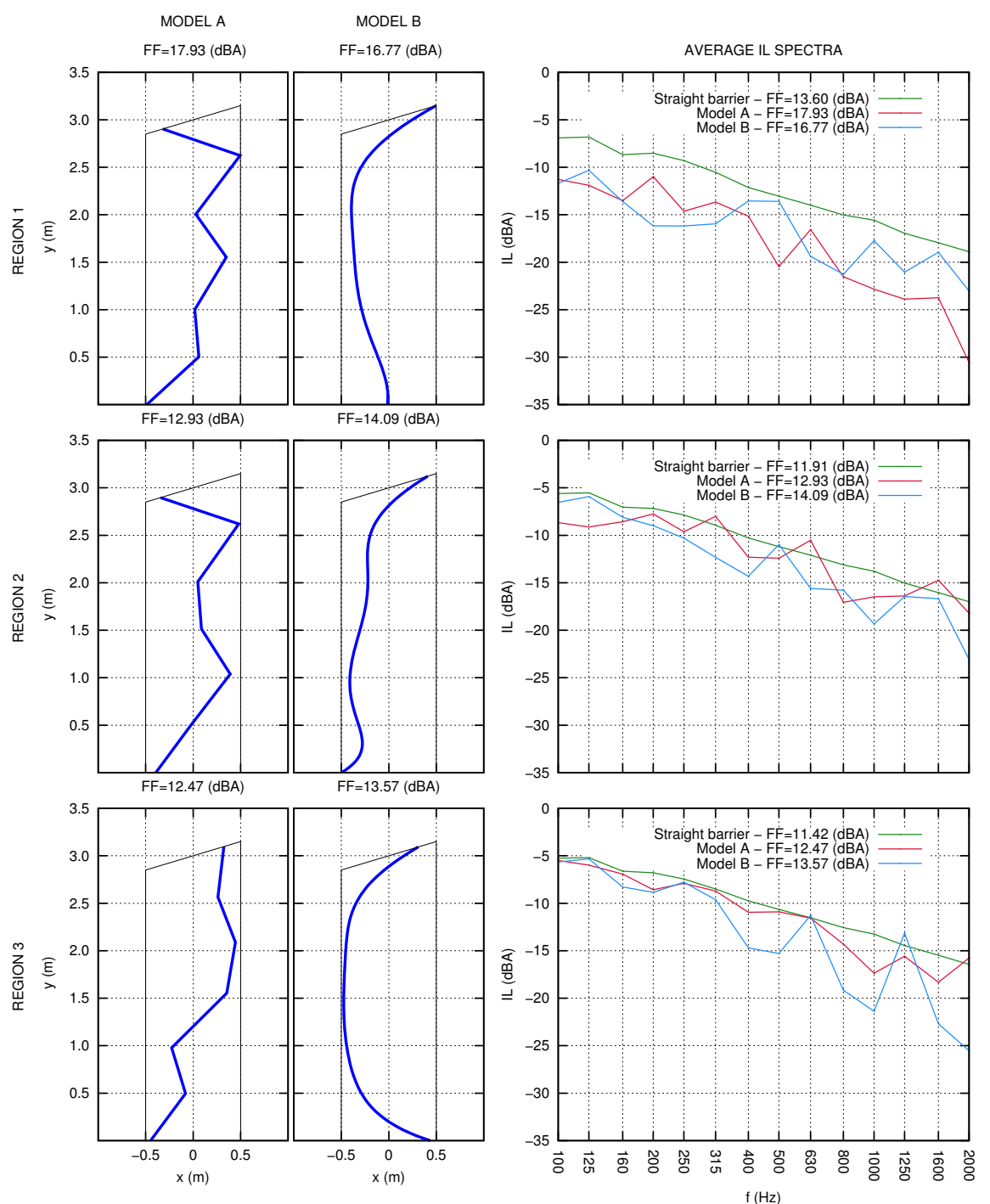

Fig. 4 Ca receiver configuration. Left, barrier profile of the best individuals for each region and model. Right, average frequential IL evolution for models A and B and for the $3 \mathrm{~m}$ height straight barrier. 

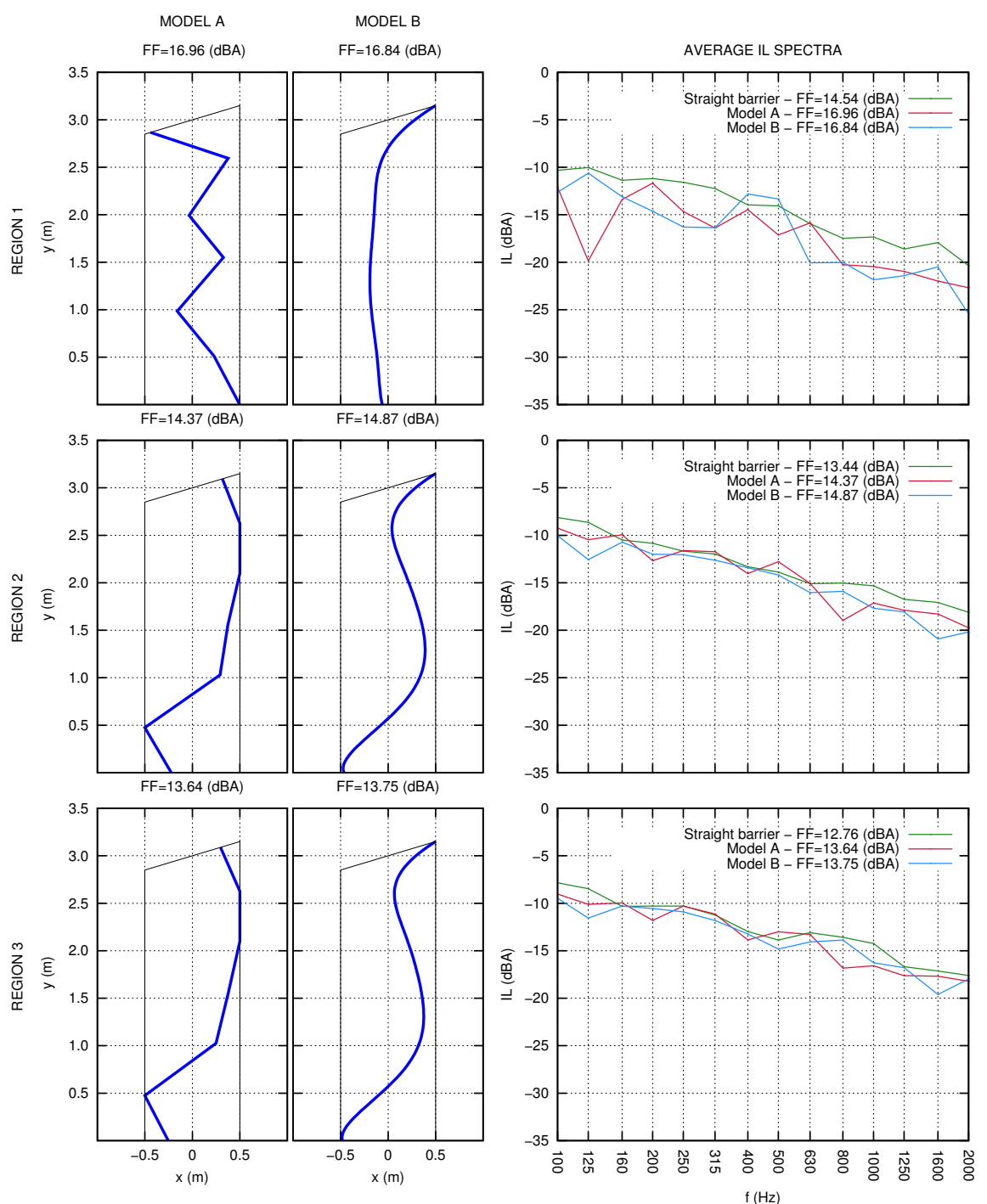

Fig. $5 \mathrm{Cb}$ receiver configuration. Left, barrier profile of the best individuals for each region and model. Right, average frequential IL evolution for models A and B and for the $3 \mathrm{~m}$ height straight barrier. 


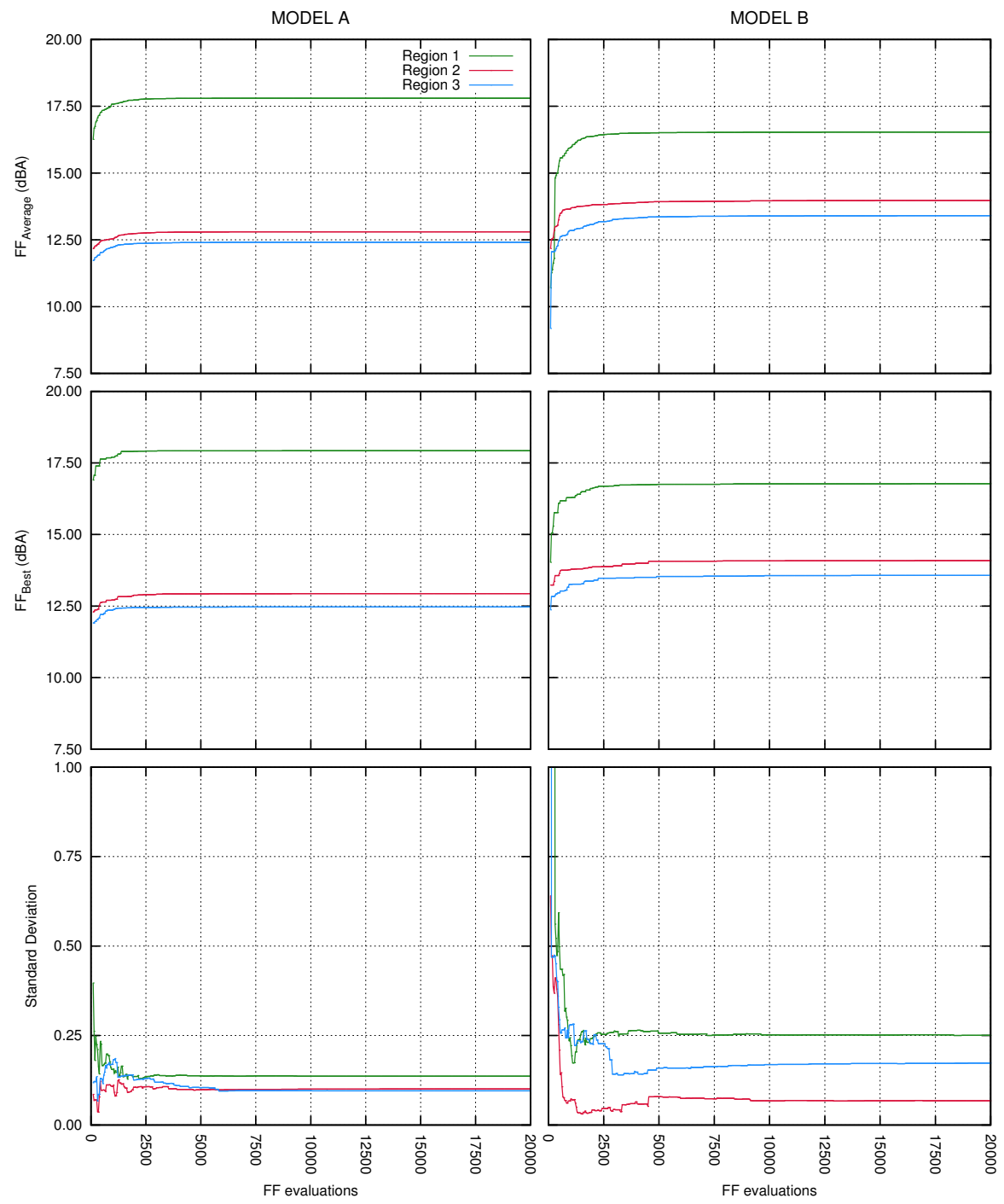

Fig. 6 Ca receiver configuration. Left, model A. Right, model B. 


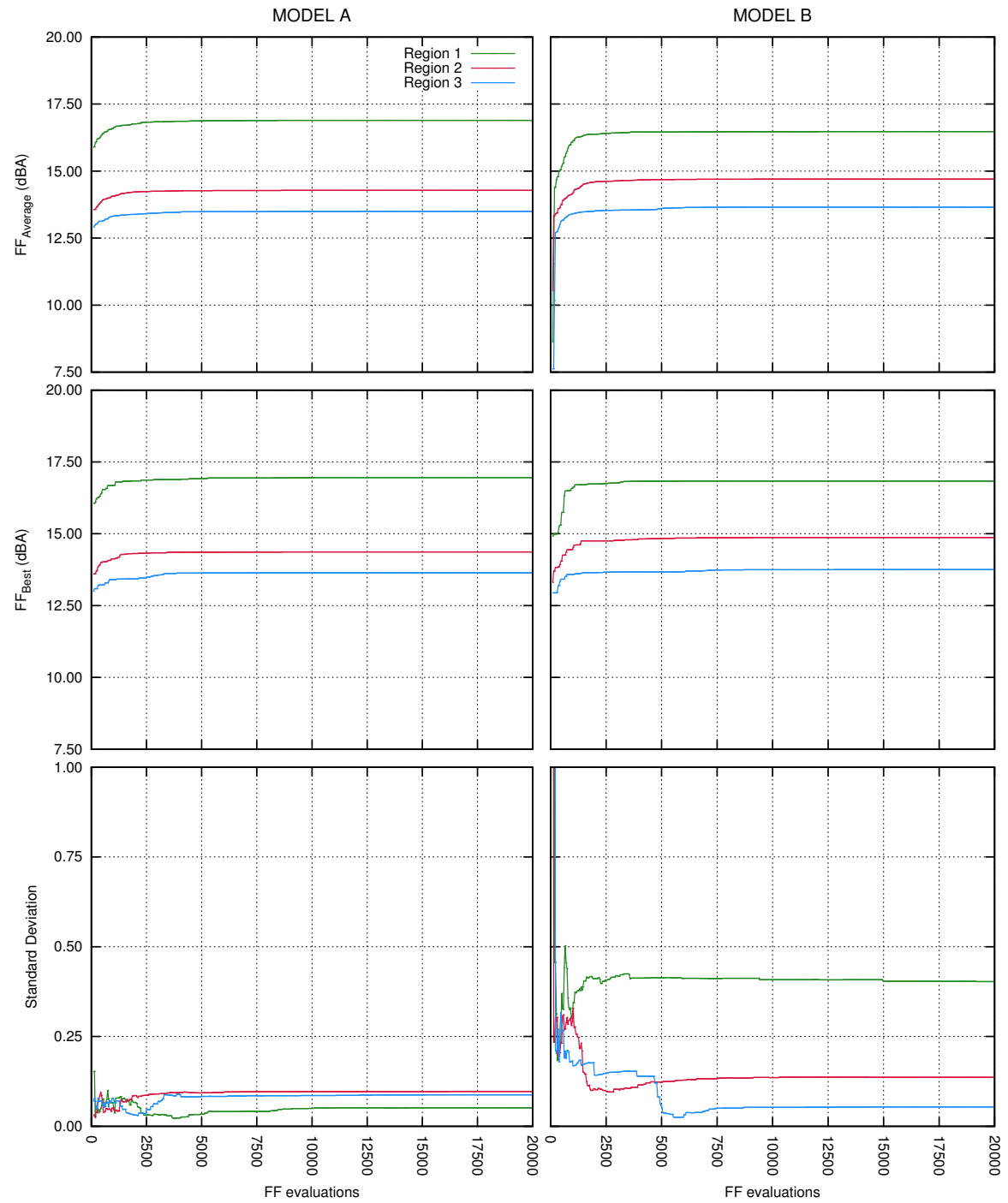

Fig. $7 \mathrm{Cb}$ receiver configuration. Left, model A. Right, model B. 


\section{Conclusions}

A methodology to successfully optimize thin cross-section noise barriers by idealizing their profiles as null cross-section boundaries has been presented. This procedure has been applied to two specific noise barrier models although its applicability covers a wide designs spectra, ranging from complex straight boundary configurations to curve-shaped profiles like those built from Spline expressions, amongst others.

The versatility of the algorithm responsible for the geometry generation of the barrier makes the building of the profile to be easily accomplished. This is a significant advantage over the case when dealing with geometries of real barrier profiles, as the evaluation process for the feasibility of the design proposed by the EA is often complex and difficult to establish.

The procedure here presented is a useful method to assess the behaviour of complex noise barriers configurations and yields conclusions that might have been hardly drawn without its implementation.

Acknowledgements This work was supported by the Ministerio de Economía y Competitividad (MINECO) of Spain and FEDER through research project BIA2010-21399-C02-01 and also by the Agencia Canaria de Inv., Inn. y Soc.de la Inf.(ACIISI) of the Government of the Canary Islands and FEDER through research project ProID20100224. R. Toledo is a recipient of a fellowship from the Subprogram of Predoctoral Fellowships of Research Personnel in Trainning (FPI-MICINN), granted by Ministerio de Ciencia e Innovación of Spain. The authors are grateful for this support.

\section{References}

1. ISO 717.2 - Rating of sound insulation in buildings and of building elements - Part 2: Impact sound insulation

2. Greiner, D., Aznárez, J., Maeso, O., Winter, G.: Shape design of noise barriers using Evolutionary Optimization and Boundary Elements. The Fifth International Conference on Engineering Computacional Technology, Civil-Comp-Press, Stirlingshire, U.K. 43 (2006)

3. Greiner, D., Aznárez, J.J., Maeso, O., Winter, G.: Single- and multi-objective shape design of Y-noise barriers using Evolutionary computation and Boundary Elements. Advances in Engineering Software, Elsevier 41(2), 368-378 (2010)

4. Greiner, D., Galván, B., Aznárez, J., Maeso, O., Winter, G.: Robust design of noise attenuation barriers with Evolutionary multiobjective algorithms and the Boundary Element Method. LNCS, Evolutionary Multi-Criterion Optimization 5467, 261-274 (2009)

5. de Lacerda, L., Wrobel, L., Mansur, W.: A dual boundary element formulation for sound porpagation around barriers over an impedance plane. JSV 202(2), 235-247 (1997)

6. Maeso, O., Aznárez, J.J.: Estrategias para la reducción del impacto acústico en el entorno de carreteras. Una aplicación del Método de los Elementos de Contorno. Universidad de Las Palmas de Gran Canaria. ISBN: 84-689-0340-X (2005). DOI 846890340X

7. Sáez, A., Gallego, R., Domínguez, J.: Hypersingular quarter-point boundary elements for crack problems. International Journal for Numerical Methods in Engineering 38, 1681-1701 (1995)

8. Toledo, R., Aznárez, J.J., Maeso, O., Greiner, D.: Optimización de diseños de pantallas acústicas de pequeño espesor mediante la implementación de la formulación dual del mec. In: Congress on Numerial Method in Engineering, SEMNI (2013)

9. Wall, M. (ed.): GAlib: A C++ Library of Genetic Algorithm Components (http://lancet.mit.edu/ga/). Mechanical Engineering Department, MIT (1996) 